magnetic susceptibility changes from $-0.200 \times 10^{-6}$ to $-0 \cdot 140 \times 10^{-6}$. From the course of the curve, it might be expected that at an extremely high stress (density $=10.365$ ) the susceptibility of cold-worked

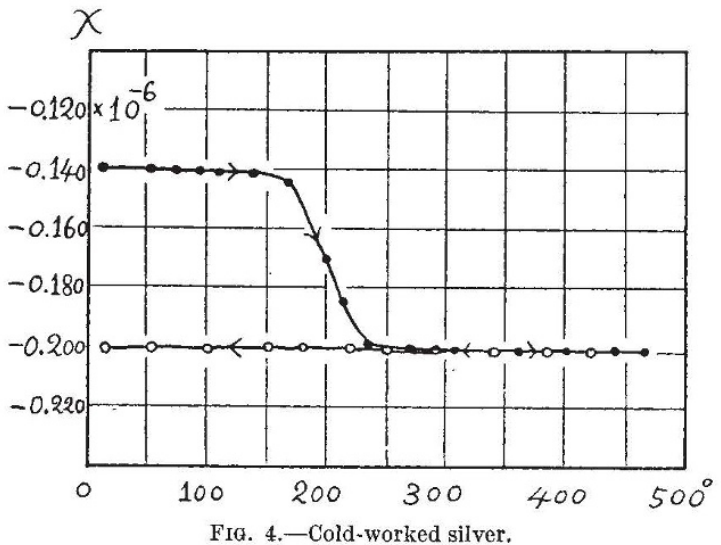

silver will change from the diamagnetic to a paramagnetic. The annealing experiment (Fig. 4) shows also that the change is a true one.

Thus we have now two examples, in which the magnetic susceptibility of a substance changes its sign with external conditions, namely, tin and copper; the former (l.c., p. 134) changes its sign with temperature and the latter with internal stress.

According to Honda's theory of magnetism (l.c., p. 185), the magnetic susceptibility of a substance is the sum of a paramagnetic term $\chi_{p}$ and a diamagnetic one $\chi_{d}$, that is,

$$
\chi=\chi_{p}-\chi_{a} .
$$

By applying a stress to a metal its density diminishes, and this will cause an increase of $\chi_{p}$ by the decrease of mutual action among neighbouring atoms (l.c., p. 181), and also an increase of $\chi_{d}$ by an increase of bound electrons (l.c., p. 186) at the cost of free electrons, so that in the case of diamagnetic metals the difference $-\left(\chi_{d}-\chi_{p}\right)$ may numerically decrease as in the case of silver, and may even become positive as in the case of copper, provided that the rate of increase of $\chi_{p}$ is greater than that of $\chi_{d}$.

KotaRô HoNDA.

Yosomatsu Shimizo.

Research Institute for Iron, Steel, and Other Metals,

Sendai, Japan, Oct. 27.

\section{Effect of a Direct Current on the Frequency of a Sonometer Wire.}

IN a communication in NATURE of May 31, p. 819 , Messrs. D. V. Gogate and Y. G. Naik describe an experiment showing that a vibrating sonometer wire undergoes a lowering in frequency when placed in a D.C. circuit. They affirmed that this unexpected result was due neither to the heating of the wire nor to a magnetic action, but at the time offered no explanation of the cause. In their experiment a sonometer wire was tuned to the frequency of an electrically driven fork so that synchronous vibrations of a large amplitude were set up in the wire. When a direct current was sent through it, the amplitude was immediately reduced, and could be restored again to its original width by shortening a little the distance between the bridges, thus showing that the frequency of the wire had been lowered.

This seemingly inexplicable phenomenon aroused some interest in this laboratory, and at the suggestion of Dr. S. J. M. Allen a detailed investigation was carried out. It was found that there is nothing mysterious about the effect. It is simply the result of a decrease of tension in the vibrating portion of the wire due to thermal expansion and the friction at the bridge. This explanation can be verified by the following experiments :

(1) The experiment of Messrs. Gogate and Naik was repeated and similar results were observed.

(2) To test the effect of heating on the wire, a still wire was strung parallel and close to it. When a current was sent through this still wire, a decrease in amplitude of the vibrating wire, similar to that of the previous experiment, was observed. However, when a sheet of mica was interposed between the two so as to cut off immediate heat transfer, the passage of a current through the still wire produced no change in amplitude. The effect of heating was further tested by holding a hot rod close to the vibrating wire. A change in amplitude and accompanying change in frequency, similar to that produced by the current, was at once observed, thus showing that the change in frequency was due to heating.

(3) The fixed end of the wire was attached to a small spring balance which was firmly clamped to the first bridge. Tension was maintained by a weight and pulley. When a current was sent through the vibrat. ing wire, a displacement of the pointer on the balance, as viewed through a low-powered microscope, indicated a decrease in the tension of the vibrating portion. When the current was broken, the pointer moved back to its original position. This proves that the frequency is lowered by a decrease in tension. A curve plotted with change in tension as ordinates and current as abscissæ takes the form of a parabola, but if the square of the current is used as abscissæ the result is a straight line, showing that the change is directly proportional to the heating of the wire.

Wires of different materials were used. It was found that for those having large expansion coefficients the tension change was greater than the corresponding change for wires having small expansion coefficients; for example, the tension change for German silver, having a coefficient of 0.000018 , was almost twice as great as that for steel with a coefficient of 0.000010 . This shows that the change in tension is dependent upon the expansion of the wire.

As the wire expands and moves over the bridge its motion is opposed by the friction of the bridge so that the slack in the wire is not all taken up by the weight. The tension in the vibrating portion, then, will differ from that on the opposite side of the bridge by an amount equal to the frictional foree at the bridge.

(4) To test the effect of the bridge friction, the sonometer was placed in a vertical position and the bridge replaced by a pulley, thus reducing the friction to a minimum. Then, when a current was passed through the vibrating wire, no change in amplitude, and consequently no change in frequency, could be detected, even though large currents were passed through the wire.

Quantitative results will be published elsewhere. ROLAND ScHAFFERT

University of Cincinnati, (Laws Fellow in Physics) Nov. 13.

\section{A Biological Station for the Red Sea.}

The Red Sea is one of the most interesting in the world, and the usefulness of a biological station on its shore scarcely needs emphasis. Owing to its peculiar physical features-desert coasts and a shallow sill separating it from the Indian Oceanwarm water, with active coral growth, and, with

No. 3191, VoL. 126] 